NOTICE: this is the author's version of a work that was accepted for publication in Journal of Fluids and Structures. Changes resulting from the publishing process, such as peer review, editing, corrections, structural formatting, and other quality control mechanisms may not be reflected in this document. Changes may have been made to this work since it was submitted for publication. A definitive version was subsequently published in Journal of Fluids and Structures, 27, 3, 2011. DOI: 10.1016/j.jfluidstructs.2010.11.014 


\title{
The effect of inertial inhomogeneity on the flutter of a cantilevered flexible plate
}

\author{
Howell, R.M., Lucey, A.D. and Pitman, M.W. \\ Fluid Dynamics Research Group, Curtin University, \\ GPO Box U1987, Perth, WA 6845, Australia \\ E-mail: $\underline{\text { A.Lucey@Curtin.edu.au }}$
}

\begin{abstract}
1 ABSTRACT
2 We study the effect of adding discrete structural mass on the linear stability of an otherwise homoge3 nous cantilevered-free flexible plate immersed in uniform axial flow. The methods of Howell et al. 4 (JFS 2009, 25:544-546) that mixed numerical simulation with eigenvalue analysis are simply extended 5 for the present study. An ideal two-dimensional flow is assumed wherein the rotationality of the 6 boundary-layers is modelled by vortex elements on the solid-fluid interface and the imposition of the 7 Kutta condition at the plate's trailing edge. The Euler-Bernoulli beam model is used for the structural 8 dynamics. It is shown that addition of mass to the plate can be either stabilising or destabilising, 9 depending upon the location of the added mass, and how its inclusion modifies the energy exchanges of the corresponding homogeneous structure. Our results therefore suggest a straightforward means by which the critical flow speed at which low-amplitude flutter sets in can be passively controlled in 2 engineering applications.
\end{abstract}

Keywords: Fluid-structure interaction, flexible surface, flutter instability, modal analysis, computational modelling

Brief Communication submitted to Journal of Fluids and Structures, December 2009

\section{${ }_{16} 1$ INTRODUCTION}

This brief communication reports the effect of adding localised structural mass on the linear flutter bounds of a thin flexible plate that is exactly aligned with the direction of a uniform flow. A schematic of the two-dimensional system studied is depicted in Fig. 1. The added mass is assumed to be a line element of dense material that does not contribute to the flexural rigidity of the plate nor does it interfere with the fluid flow; for the latter assumption to hold perfectly the added mass would be 
embedded within the plate material. Gravitational effects are neglected and thus in the unperturbed system state the plate is aligned with the $\mathrm{x}$-axis. When subjected to a small-amplitude perturbation, the contour of the deformed plate causes it to become a lifting surface over which a non-uniform fluid pressure field acts. This further deforms the plate which, in turn, modifies the lift force and its associated pressure field. Thus, a fluid-structure interaction is established that may lead to either attenuating, neutrally-stable or amplifying oscillations of the structure. Of particular importance is the critical value of the flow speed at which fluid-loaded vibration first become unstable. While this system is of fundamental interest, its dynamics may be relevant to many physical systems ranging from fluttering flags, through to oscillations of the human soft-palate that create snoring noises, and to energy-harvesting devices that could extract fluid energy through its transfer to the plate in a process of controlled destabilisation.

Kornecki et al. (1976) were the first to conduct comprehensive modelling and analysis of the problem at hand, although it has classical roots that date back to Lord Rayleigh and see, for example, the elegant experiments of Zhang et al. (2000) who investigated the oscillation of a filament in a soap-film flow. Using ideal flow Kornecki et al. studied the two-dimensional problem of flexible plate embedded in an infinite domain of fluid, as did the more recent work of Huang (1995), Yamaguchi et al. (2000), Watanabe et al. (2002), Argentina \& Mahadevan (2005) and Tang \& Päidoussis (2007). Eloy et al. (2007, 2008) incorporated the effects of finite aspect ratio showing that these principally served as a correction to the fundamentally two-dimensional dynamics of the problem. The flow-plate configuration has been extended to that of a flexible plate mounted in plane-channel flow; see Auregan \& Dépollier (1995) and Guo \& Paidoussis (2000). All of these studies predict that the plate loses its stability through flutter that sets in beyond a critical uniform flow speed or Reynolds number in the case of viscous channel flow; for the latter refinements see Balint \& Lucey (2005) and Tetlow \& Lucey (2009). For short plates the flutter mode is predicted to comprise mainly a combination of the first and second in-vacuo modes while for long plates, or plates with heavy fluid loading, the critical mode is dominated by higher-order mode content. The recent work of Howell et al. (2009) elucidated the instability mechanisms showing that 'short' plates succumb to single-mode flutter while 'long' plates are destabilised by modal-coalescence flutter of the Kelvin-Helmholtz type predicted exactly for fluidloaded plates of infinite extent and discussed, for example, by Crighton \& Oswell (1991) and Lucey (1998).

In this brief communication we extend the work of Howell et al. (2009) to model and investigate the effect of adding localised mass for a range of values and at different locations along the flexible plate. Certain counter-intuitive results are obtained; for example, the addition of mass can be stabilising and these are explained in terms of the energy transfers between the fluid and the plate. These may be of particular relevance in the design of the flutter mill recently proposed and studied by Tang et 
al. (2009) wherein instability of a flexibility plate is used to harvest the kinetic energy of the mean flow.

\section{THEORETICAL AND COMPUTATIONAL MODELS}

The essential modelling is described in detail in Howell et al. (2009) wherein the system of Fig. 1 was mounted symmetrically within a channel with its walls located at $y= \pm H$; the present system is obtained by letting $H \rightarrow \infty$. We also neglect the effects of the wake that were modelled in the precursor paper because these were shown to be significant only for very short panels; we will return to this below.

For ease of reading, we first summarise the approach of Howell et al. (2009) as applied to the present investigation and then show how inertial inhomogeneity is readily incorporated in the model. The flow field is found using a linearised boundary-element method (BEM) with first-order vortex panels on the flexible plate because of the discontinuity of tangential fluid velocity across this surface that makes it a lifting surface; the distributed lift drives the motion of the flexible plate. The singularity strengths are determined by enforcing the no-flux boundary condition at every panel control point and continuity of the distributed vorticity between adjacent panels used to discretise the flexible plate. Thus the vector of singularity strengths is given by

$$
\left\{\Gamma_{m}\right\}=\left[I_{i m}^{N}\right]^{-1}\left\{U_{\infty} \theta_{m}+\dot{\eta}_{m}\right\}
$$

where $\Gamma_{m}$ contains the zero- and first-order order coefficients of the singularity distributions on the panels in the BEM. $\left[I_{i m}^{N}\right]^{-1}$ contains, in addition to the normal influence-coefficients of the singularities, the boundary conditions of: a) vortex strength continuity at panel end points; and b) zero vorticity at the plate's trailing edge, thus enforcing the standard Kutta condition of zero pressure difference at the trailing edge for linear displacements. $\theta_{m}$ is the panel's angle to the horizontal which in the linear framework is the streamwise spatial derivative of the boundary, $\eta_{m}$.

The unsteady Bernoulli equation is used to determine the pressure distribution on the flexible plate. The transmural pressure is then used as the forcing term in the one-dimensional thin flexibleplate equation couched in finite-difference form. The motions of the plate and the fluid flow are fully coupled through deflection, vertical velocity and acceleration of the two media at their interface. This allows the following single system (matrix) equation to be written

$$
\begin{aligned}
\rho h[\mathbf{I}]\left\{\ddot{\eta}_{m}\right\}+d[\mathbf{I}]\left\{\dot{\eta}_{m}\right\}+B\left[\mathbf{D}_{\mathbf{4}}\right]\left\{\eta_{m}\right\} & =2 \rho_{f} U_{\infty}^{2}(1 / \delta x)\left[\mathbf{B}_{\mathbf{1}}^{+}\right]\left\{\eta_{m}\right\} \\
& +\rho_{f} U_{\infty}(1 / \delta x)\left[\mathbf{B}_{\mathbf{2}}^{+}\right]\left\{\dot{\eta}_{m}\right\}+\rho_{f} U_{\infty}\left[\mathbf{B}_{\mathbf{1}}^{-}\right]\left\{\dot{\eta}_{m}\right\} \\
& +\rho_{f}\left[\mathbf{B}_{\mathbf{2}}\right]\left\{\ddot{\eta}_{m}\right\},
\end{aligned}
$$

where $[\mathbf{B}]$ are matrices of singularity influence coefficients, $\left[\mathbf{D}_{4}\right]$ is a fourth-order spatial-differentiation 
matrix and $[\mathbf{I}]$ is the identity matrix. $\rho, h, d$ and $B$ are respectively, the material density, thickness, dashpot-type damping coefficient and flexural rigidity of the plate, the dynamics of which appear on the left-hand side of the equation. Uniform discretisation of the plate length $L$ into $N$ collocation points defines $\delta x=L / N$. The pressure perturbation that drives the plate motion appears on the right-hand side, where $\rho_{f}$ and $U_{\infty}$ are the fluid density and flow speed. The pressure terms in the line order of Eqn. 2 can be interpreted as the hydrodynamic stiffness, damping and inertia respectively.

The formulation of Eqn. 2 is for a homogeneous flexible plate; Howell et al. (2009) briefly investigated the inhomogeneous case of spatially varying flexural rigidity, $B$. In the present study, we model inertial inhomogeneity by introducing a point mass at a specific plate location $\bar{x}_{p}$ (where $\left.0<x_{p}<L\right)$; its value is given in quanta, $n^{+}$, of total plate mass (per unit width), $M^{T}=\rho h L$. To effect this, the element $(m, m)$ of the identity matrix, $[\mathbf{I}]$ in Eqn. 2 becomes $\left(1+n^{+}\left(M^{T} / \delta x\right)\right)$ where $m=\operatorname{int}\left[\left(x_{p} / L\right) N\right]$ is the collocation point closest to the location at which the point mass is added.

We take two approaches to the solution of Eqn. 2. In the first we reduce the second-order ordinary differential equation in $\{\eta\}$ to first-order using the state-space variables $w_{1}(t)=\eta(t)$ and $w_{2}(t)=\dot{\eta}(t)$.

Rearranging in companion-matrix form then yields the system equation

$$
\dot{w}=[H] w, \quad \text { where } \quad w=\left\{w_{1}, w_{2}\right\}^{T} .
$$

Single-frequency time-dependent response is assumed at $\omega$ which is a complex eigenvalue of $[H]$. Positive $\omega_{I}$ and $\omega_{R}$ respectively represent the oscillatory and amplifying parts of the response.

Alternatively, we perform a time-discretisation of Eqn. 2 and then numerically time-step, using a semi-implicit method, the equation to determine the system response to some form of initial perturbation. In doing so we are able to study transient behaviour and reveal localised flow-structure dynamics that when summed contribute to the system response.

\section{RESULTS AND DISCUSSION}

All of our results are presented in non-dimensional form having used the scheme of Crighton \& Oswell (1991), and derived more explicitly in Lucey et al. (2003), whereby

$$
\bar{t}=t\left[\rho_{f}^{2} B^{\frac{1}{2}} /(\rho h)^{\frac{5}{2}}\right], \quad \bar{U}=U_{\infty}\left[(\rho h)^{\frac{3}{2}} /\left(\rho_{f} B^{\frac{1}{2}}\right)\right] \quad \text { and } \quad \bar{d}=d\left[(\rho h)^{\frac{3}{2}} /\left(\rho_{f}^{2} B^{\frac{1}{2}}\right)\right] . \quad(4 \mathrm{a}, \mathrm{b}, \mathrm{c})
$$

The non-dimensional streamwise coordinate, the length (or mass ratio) of the flexible plate and the channel height are defined by

$$
\bar{x}=x / L, \quad \bar{L}=L\left[\rho_{f} /(\rho h)\right] \text { and } \bar{H}=H[1 / L] . \quad(5 \mathrm{a}, \mathrm{b}, \mathrm{c})
$$


This scheme permits $\bar{U}$ and $\bar{L}$ to be interpreted respectively as the physical flow speed and plate length for given fluid and plate properties.

Howell et al. (2009) showed that when channel walls were present at $y= \pm H$ their effect was negligible for $\bar{H}=1$; i.e. the fluid-structure system behaved as if it were in an infinite domain of fluid. We also use a perfectly elastic plate, hence $\bar{d}=0$, in the present study. Howell et al. (2009) presented the effects of channel wall proximity, damping and an upstream splitter plate on the fundamental system studied here; the reader is therefore referred to that work for the details of these refinements. We discretise the flexible plate into $N=50$ panels, following Howell et al. (2009) wherein the present methods were validated, and extract all 50 system eigenmodes. In summary, the non-dimensional control parameters of the system investigated are the flow speed $\bar{U}$, the plate length $\bar{L}$, the quantity of added mass $n^{+}$, and its location $\bar{x}_{p}$.

To understand the results of our numerical simulations, we consider the energy evolution of the system. Adapting the derivation of Balint \& Lucey (2005), the plate energy changes with time according to

$$
\frac{d}{d t}(\underbrace{\frac{1}{2} \rho h \int_{0}^{L} \dot{\eta}^{2} d x}_{E_{k}}+\underbrace{\left.\frac{1}{2} n^{+} M^{T} \dot{\eta}^{2}\right|_{x=x_{p}}}_{E_{k}^{+}}+\underbrace{\frac{1}{2} B \int_{0}^{L} \eta_{, x x}^{2} d x}_{E_{s}})=\underbrace{\int_{0}^{L}(-\Delta p) \dot{\eta} d x}_{\dot{W}},
$$

where the total plate energy, $E_{t}$, is the sum of the kinetic energies of plate, $E_{k}$, and added mass, $E_{k}^{+}$, plus the plate's strain energy, $E_{s}$. Equation 6 shows that the plate energy either grows or decays in time depending upon the rate of work done by the pressure loading, $(-\Delta p)$ (determined by the right-hand side of Eqn. 2). If structural damping were present, then a further energy-dissipation term appears on the right-hand side; see Balint and Lucey (2005) and Howell et al. (2009) for a discussion of its effect. In our results we present energy records, $\bar{W}(t)$, that represent the sum of pressure work done up to time $t$ and therefore corresponds to the current value of total plate energy $E_{t}$; these are non-dimensionalised by the initial strain energy of the plate due to the applied deflection.

\section{1 'Short' plates - low mass ratio}

We first review the results for a homogenous, or 'standard', short plate at $\bar{L}=1$ presented in Howell et al. (2009) for $\bar{H}=1$. Figure 2a shows the variation of system eigenvalues with applied flow speed. Single-mode flutter of the second system mode is the critical instability at a non-dimensional flow speed $\bar{U}=\bar{U}_{c}=5.452$. Figure $2 \mathrm{~b}$ shows the numerical simulation of the critical mode at this flow speed. The simulation was started by releasing the plate from an applied deformation - the thick black line - in the shape of the second in-vacuo mode. The critical mode, seen to contain strong contributions from the first and second in-vacuo modes, then evolves from the initial excitation. Howell et al. monitored the phase angle between the pressure loading and plate velocity in numerical simulations 
showing that both the leading-edge singularity and the trailing-edge Kutta condition contribute to non-orthogonality of the pressure and plate velocity. The product of these terms yields the localised rate of work done (per unit area of plate) which is then non-zero when integrated over one period of oscillation. The plate-energy record for the simulation of Fig. 2b is shown in Fig. 2c. After transients due to mode adjustment have convected away, the total energy is constant. However, it is also seen that the energy exchanges between plate and flow are spatially dependent; thus for example, the third quarter from the leading edge of the flexible plate receives energy while the fourth quarter gives energy to the fluid flow. It is the sum of all the spatially dependent energy transfers that, in this case, yields the neutral stability of the system at this critical speed.

We now show how the addition of a point mass changes the results of the standard case characterised by Fig. 2. Figures 3 and 4 show the effect of adding a point mass of $n^{+}=3$ (three plate masses) at positions $\bar{x}_{p}=0.625$ and $\bar{x}_{p}=0.875$ respectively. In each figure the mode and energy plots are at the critical flow speed. Adding the mass at $\bar{x}_{p}=0.625$ is stabilising because it increases $\bar{U}_{c}$ to 6.609 . This occurs because of the reduced energy transfer to the plate in the third quarter that principally drives single-mode flutter; see Fig. 2c. The energy transfer to the plate in its second quarter is increased and this is a typical feature of modal-coalescence flutter as will be seen in $\S 3.2$. However, this additional source of destabilisation is offset by an increased stabilising energy transfer to the fluid in the fourth quarter of the plate. The eigenvalue plot of Fig. 3a shows that the mass addition causes coupling between Modes 1 and 2 and this interrupts the single-mode flutter mechanism of the standard case. This effect is clearly seen in the mode plot of Fig. 3b that strongly features a Mode-1 contribution whereas the homogeneous case of Fig. 2b is dominated by Mode 2 content. Thus, the addition of the mass effectively replaces the critical single-mode flutter mechanism with that of modal-coalescence.

In contrast, the same point mass added at $\bar{x}_{p}=0.875$ is destabilising with the critical flow speed reducing to 4.406 from the 5.452 of the standard case. The energy record of Fig. 4c shows that the added mass interrupts the stabilising effect of the fourth quarter of the plate that in the standard case of Fig. 2c transfers energy from plate to the fluid. However, the instability mechanism - single mode flutter - is unchanged as is evidenced by comparing the morphology of the corresponding eigenvalue plots, Figs. 2a and 4a. The critical mode plotted in Fig. $4 \mathrm{~b}$ is more strongly dominated by Mode 2 content as compared with Fig. 2b; this is evidence of a more potent single-mode flutter mechanism.

Figure 5 summarises the effect of adding a point mass for the case $\bar{L}=1$. Figure 5 a shows the variation of critical flow speed with the amount of mass, $n^{+}$, added at each of the positions $\bar{x}_{p}=0.375$, 0.625 and 0.875 . Mass addition at $\bar{x}_{p}=0.625$ yields modal-coalescence flutter (m-c), as shown in Fig. $3 \mathrm{a}$, and is stabilising throughout the range of $n^{+}$studied, whereas adding mass at $\bar{x}_{p}=0.375$ and 0.875 promotes, for the latter as demonstrated through Fig. 4, the single-mode flutter (smf) that is 
the critical instability for the homogenous plate. Figure 5b shows how the critical speed varies with the position, $\bar{x}_{p}$, at which the added mass is located on the plate for three different quanta, $n^{+}=1$, 2 and 3. Note that we limit mass addition to a value of $n^{+}=3$ (three times the plate mass) because to exceed this would be physically implausible. The features for the different amounts of added mass are similar. This demonstrates that its effect is critically dependent upon the location at which it is added and therefore adding mass can be used as a strategy to either increase or decrease the critical flow speed.

\section{2 'Long' plates - high mass ratio}

Howell et al. (2009) showed that homogeneous plates with high $\bar{L}$ are destabilised by modal-coalescence flutter as opposed to single-mode flutter that gives the critical condition for plates with low $\bar{L}$. Figure 6, adapted from Howell et al. (2009) for $\bar{H}=1$, shows results for a homogeneous plate corresponding to those of Fig. 2 but with $\bar{L}=10$. Instability is caused by the coalescence of Modes 2 and 3 as evidenced by Figs. 5a and 5b. The energy plot of Fig. 5c shows that the main destabilising energy transfer occurs in the second quarter of the plate. This is in contrast to the dominance of the third-quarter in providing the destabilising energy transfer of single-mode flutter as shown in $\S 3.1$.

Adding a point mass with $n^{+}=2$ at locations $\bar{x}_{p}=0.625$ and $\bar{x}_{p}=0.875$ gives the results of Figs. 7 and 8 respectively. At both locations the effect is destabilising, the critical speed dropping from 0.63 to 0.47 and 0.21 respectively. Thus, the greatest effect occurs when the added mass is placed nearest to the trailing edge of the plate. However, the increased instability of the system occurs through very different mechanisms for these two cases.

Figure 7, when compared with the corresponding results of Fig. 6, shows that the modalcoalescence mechanism continues to yield the critical speed and, indeed, is promoted because the added mass reduces the difference between oscillatory frequencies of the interacting Modes 2 and 3 . The critical mode shape and energy budgets are similar to those of the homogeneous case except that energy transfer in the third quarter of the plate becomes a marginal contributor to unstable behaviour.

When the same mass is added nearer the trailing edge of the plate at $\bar{x}_{p}=0.875$, Fig. 8 shows that single-mode flutter becomes the critical instability. This is evidenced by the critical mode of Fig. $8 \mathrm{~b}$ and the fact that the main destabilising energy transfer occurs in the third quarter of the plate as seen in Fig. 8c; both of these features bear similarity to the typical single-mode flutter results for a homogenous short plate presented in Figs. $2 \mathrm{~b}$ and 2c. The effect of the added mass at this location is to increase the difference between the oscillation frequencies of Modes 2 and 3, seen most notably at $\bar{U}=0$ in Fig. 8a. This sufficiently decouples Modes 2 and 3 so that modal-coalescence does not occur and instead Mode 2 succumbs to single-mode flutter at the lower flow speed associated with 
that destabilisation mechanism.

For $\bar{L}=10$ the effect of different amounts of added mass and its location are quantified in Fig. 9. Throughout the range of $n^{+}$and $\bar{x}_{p}$ investigated the addition of mass is seen to be destabilising. The kinks in Figs. 9a and 9b for $n^{+}=1$ and $\bar{x}_{p}=0.875$ are accounted for by the switch from a modal-coalescence flutter mechanism to that of single-mode flutter characterised by the results and discussion of Fig. 8.

\section{CONCLUDING REMARKS}

We have presented a simple adaptation of the model of Howell et al. (2009) that enables the effect of inertial inhomogeneity on the linear stability of flexible plate in a uniform flow to be studied through both an eigen-analysis and numerical simulation. Our results show that the addition of mass at a discrete location can be either stabilising or destabilising for short plates (or low mass ratios) whereas it is always destabilising for long plates (or high mass ratios). These results can be understood in the destabilisation framework of Howell et al. They showed that the finiteness effects embodied in the leading-edge singularity and the Kutta condition cause short plates to be destabilised by single-mode flutter whereas long plates succumb to modal-coalescence flutter brought about by the relative (to structural forces) magnitude of the fluid loading at higher flow speeds. For short plates the present study shows that the addition of mass can promote modal coupling which causes the single-mode flutter to be replaced by modal-coalescence flutter as the critical instability at a higher critical speed. Alternatively, the single-mode flutter mechanism that is critical for homogeneous short plates can be promoted leading to a lower critical speed. Which of these two effects occurs depends critically upon the location of the added mass. In contrast, long homogenous plates are only destabilised by the addition of mass because either the modal-coalescence mechanism is promoted or because it can be replaced by single-mode flutter giving a much lower critical flow speed. Again, the location of the added mass determines which form of increased destabilisation occurs.

A limitation of the present study is that the effect of the wake was omitted. Howell et al. (2009) studied this via numerical simulation and broadly showed that it inhibited the single-mode flutter of short plates and promoted the modal-coalescence flutter of long plates thereby increasing the critical flow speed of the former and lowering it for the latter. The interplay between the two types of instability caused by the addition of mass is therefore likely to be quantitatively modified when wake effects are included. However it would be reasonable to speculate that those cases for which modalcoalescence flutter was the critical instability would have a slightly lower critical flow speed while the opposite would hold where added mass promoted single-mode flutter. While the methods of Howell et al. could be modified to generate numerical simulations with both inertial inhomogeneity and wake 
effects, their extension so as to permit the extraction of system eigenmodes is not so straightforward; this next step is a more complex piece of work that is underway.

\section{REFERENCES}

Argentina, M. \& Mahadevan, L. 2005 Fluid-flow-induced flutter of a flag. Proceedings of the National Academy of Sciences 102, pp. 1829-1834.

Aurégan, Y. \& Depollier, C. 1995 Snoring: Linear stability analysis and in vitro experiments. Journal of Sound and Vibration 188, pp. 39-54.

Balint, T. S. \& Lucey, A. D. 2005 Instability of a cantilevered flexible plate in viscous channel flow. Journal of Fluids and Structures 20, pp. 893-912.

Crighton, D.G. \& Oswell, J.E., 1991, Fluid loading with mean flow. I. Response of an elastic plate to localized excitation. Philosophical Transactions of the Royal Society of London A 335, pp. 557-592.

Eloy C., Lagrange R., Souilliez C. \& Scrouveiler, L. 2008 Aeroelastic instability of cantilevered flexible plates in uniform flow. Journal of Fluid Mechanics 611, pp. 97-106.

Eloy, C., Souilliez, C. \& Schouveller, L. 2007 Flutter of a rectangular plate. Journal of Fluids and Structures 23, pp. 904-919.

Guo, C. Q. \& Païdoussis, M. P. 2000 Stability of rectangular plates with free side-edges in twodimensional inviscid channel flow. Journal of Applied Mechanics 67, pp. 171-176.

Howell, R.M., Lucey, A.D., Carpenter, P.W. \& Pitman M.W. 2009. Interaction between a cantileveredfree flexible plate and ideal flow. Journal of Fluids and Structures, 25, pp. 544-566.

Huang, L. 1995 Flutter of cantilevered plates in axial flow. Journal of Fluids and Structures 9, pp. $127-147$.

Kornecki, A., Dowell, E. H. \& O'Brien, J. 1976 On the aeroelastic instability of two-dimensional panels in uniform incompressible flow. Journal of Sound and Vibration, 47, pp. 163-178.

Lucey, A.D., 1998, The excitation of waves on a flexible panel in a uniform flow. Philosophical 
Transactions of the Royal Society of London A, 356, pp. 2999-3039.

Lucey, A.D., Sen, P.K. \& Carpenter, P.W. 2003, Excitation and evolution of waves on an inhomogeneous flexible wall in a mean flow. Journal of Fluids and Structures 18, pp. 251-267.

Tetlow, G.A. \& Lucey, A.D. 2009. Motions of a cantilevered flexible plate in viscous channel flow driven by a constant pressure drop. Communications in Numerical Methods in Engineering, 25, pp. $463-482$.

Tang, L. \& Païdoussis, M.P. 2007 On the instability and the post-critical behaviour of two-dimensional cantilevered flexible plates in axial flow. Journal of Sound and Vibration 305, pp. 529-542.

Tang, L., Païdoussis, M.P. \& Jiang, J. 2009 Cantilevered flexible plates in axial flow: Energy transfer and the concept of flutter mill. Journal of Sound and Vibration 326, pp. 529-542.

Watanabe, Y., Isogai, K., Suzuki, S. \& Sugihara, M. 2002 A theoretical study of paper flutter. Journal of Fluids and Structures 16, pp. 543-560.

Yamaguchi, N., Yokota, K. \& Tsujimoto, Y. 2000 Flutter limits and behaviours of a flexible thin sheet in high speed flow - I: Analytical method for prediction of the sheet behaviour. Journal of Fluids Engineering 122, pp. 65-73.

Zhang, J., Childress, S., Libchaber, A. \& Shelley, M. 2000 Flexible filaments in a flowing soap film as a model for one-dimensional flags in a two-dimensional wind. Nature, 408, pp. 835-839. 


\section{FIGURE CAPTIONS}

Figure 1 Schematic of the flexible plate with an added mass embedded in a uniform flow.

Figure 2 System dynamics for $\bar{L}=1$ : (a) Variation of eigenvalues with flow speed (oscillatory and growth/decay parts represented by broken and full lines respectively), (b) time-sequence of instantaneous plate position at critical speed $\bar{U}_{c}=5.452$ (of Mode 2 in (a), the thick line being the initial deflection), and (c) time series of cumulative energy transferred from flow to plate in — first, - - - second, - - - third, and $\cdots$ fourth quarters of the plate while —— (thick) is the total of these. Adapted from Howell et al. (2009).

Figure 3 The effect of added mass on the fluid structure interaction at $\bar{L}=1$ : system giving Fig. 2 modified by added mass $n^{+}=3$ at $\bar{x}_{p}=0.625$ giving $\bar{U}_{c}=6.609$. The black dot on the initial deflection in (b) signifies the position where the added mass is applied. (Sub-figure titles and legends as in Fig. 2.)

Figure 4 The effect of added mass on the fluid structure interaction at $\bar{L}=1$ : system giving Fig. 2 modified by added mass $n^{+}=3$ at $\bar{x}_{p}=0.875$ giving $\bar{U}_{c}=4.406$. The black dot on the initial deflection in (b) signifies the position where the added mass is applied. (Sub-figure titles and legends as in Fig. 2.)

Figure 5 Summary of the effect of added mass for $\bar{L}=1$ : variation of critical flow speed, $\bar{U}_{c}$ with (a) $n^{+}$for mass added at $\triangle \bar{x}_{p}=0.375, \times \bar{x}_{p}=0.625$, and $\circ \bar{x}_{p}=0.875$, and (b) the location on the plate, $\bar{x}_{p}$ at which the mass is added for the values $* n^{+}=1,+n^{+}=2$, and $\square n^{+}=3$. The labels smf and m-c denote single-mode and modal coalescence flutter respectively as the type of flutter that yields the critical flow speed and the dashed line indicates its value for no added mass.

Figure 6 System dynamics for $\bar{L}=10$ : (a) Variation of eigenvalues with flow speed (oscillatory and growth/decay parts represented by broken and full lines respectively), (b) time-sequence of instantaneous plate position at critical speed $\bar{U}_{c}=0.63$ (of Mode 2 in (a), the thick line being the initial deflection), and (c) time series of cumulative energy transferred from flow to plate in — first, -o- second, - - third, and $\cdots$ fourth quarters of the plate while —— (thick) is the total of these. Adapted from Howell et al. (2009).

Figure 7 The effect of added mass on the fluid structure interaction at $\bar{L}=10$ : system giving Fig. 6 modified by added mass $n^{+}=2$ at $\bar{x}_{p}=0.625$ giving $\bar{U}_{c}=0.47$. The black dot on the initial 
deflection in (b) signifies the position where the added mass is applied. (Sub-figure titles and legends as in Fig. 6.)

Figure 8 The effect of added mass on the fluid structure interaction at $\bar{L}=10$ : system giving Fig. 6 modified by added mass $n^{+}=2$ at $\bar{x}_{p}=0.875$ giving $\bar{U}_{c}=0.21$. The black dot on the initial deflection in (b) signifies the position where the added mass is applied. (Sub-figure titles and legends as in Fig. 6.)

Figure 9 Summary of the effect of added mass for $\bar{L}=10$ : variation of critical flow speed, $\bar{U}_{c}$ with (a) $n^{+}$for mass added at $\triangle \bar{x}_{p}=0.375, \times \bar{x}_{p}=0.625$, and $\circ \bar{x}_{p}=0.875$, and (b) the location on the plate, $\bar{x}_{p}$ at which the mass is added for the values $* n^{+}=1,+n^{+}=2$, and $\square n^{+}=3$. The labels smf and m-c denote single-mode and modal coalescence flutter respectively as the type of flutter that yields the critical flow speed and the dashed line indicates its value for no added mass. 


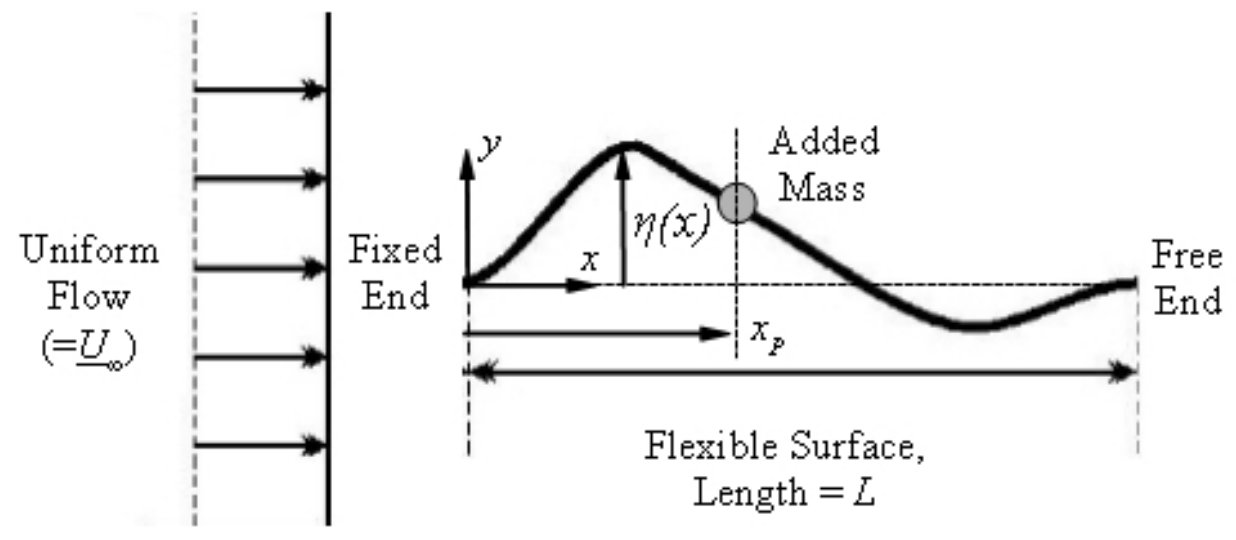

Figure 1: 


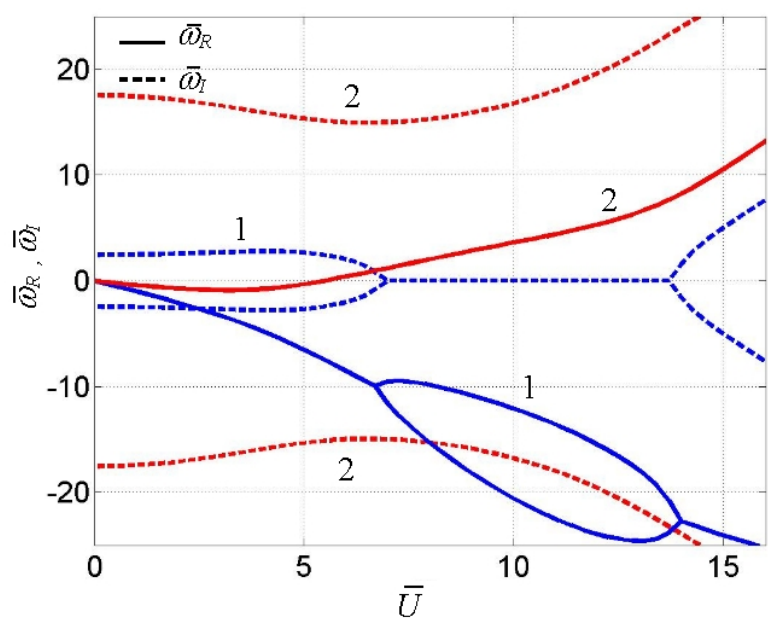

(a)

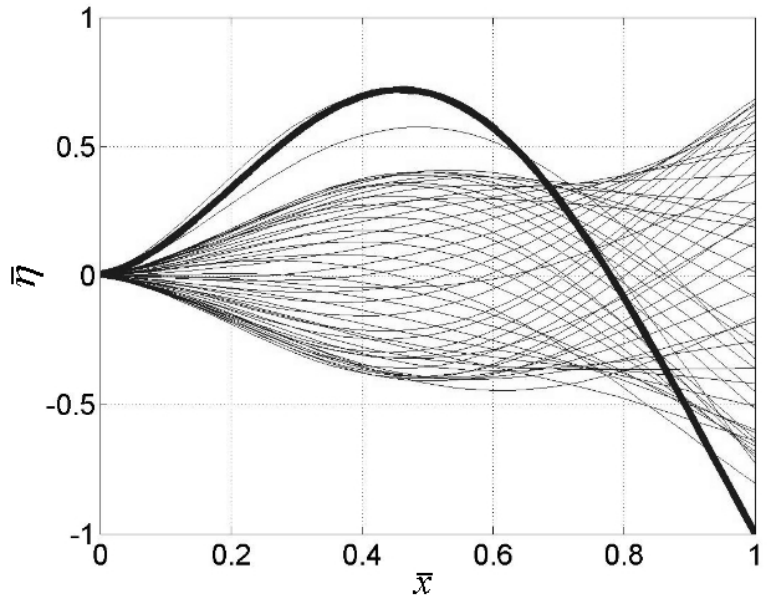

(b)

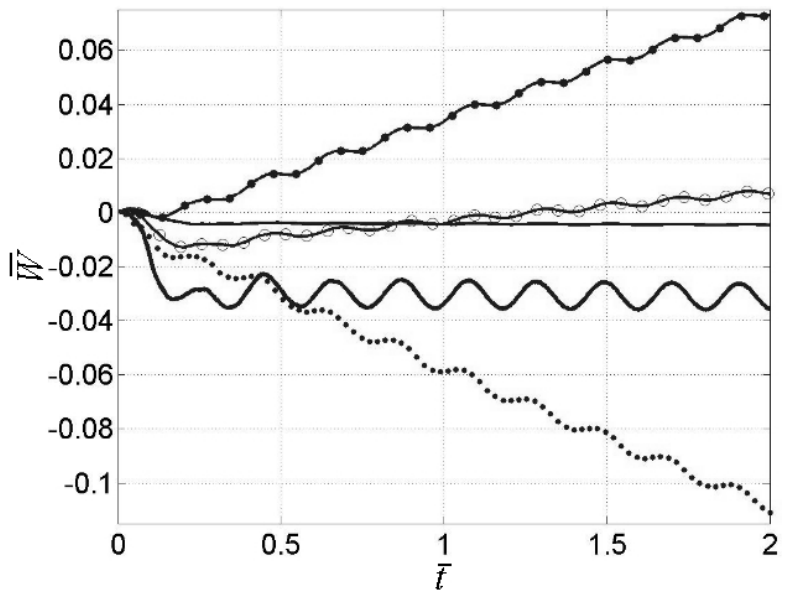

(c)

Figure 2: 


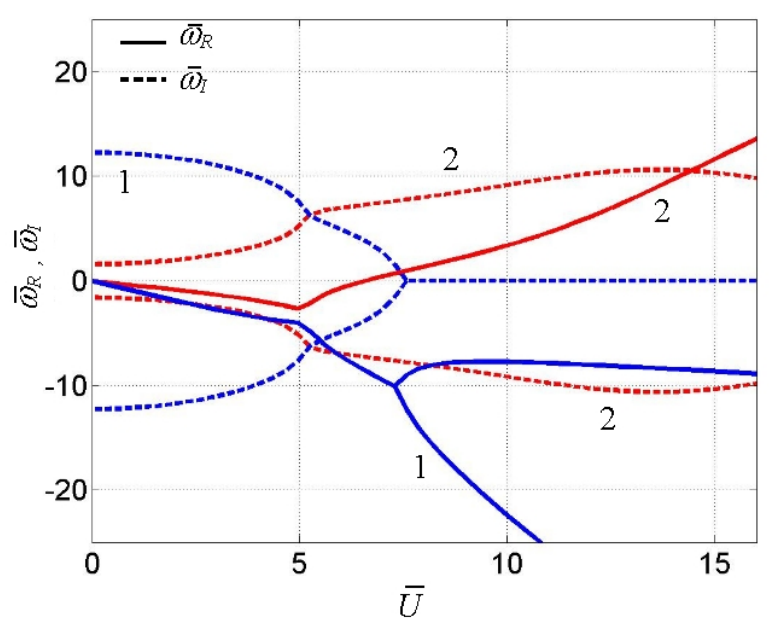

(a)

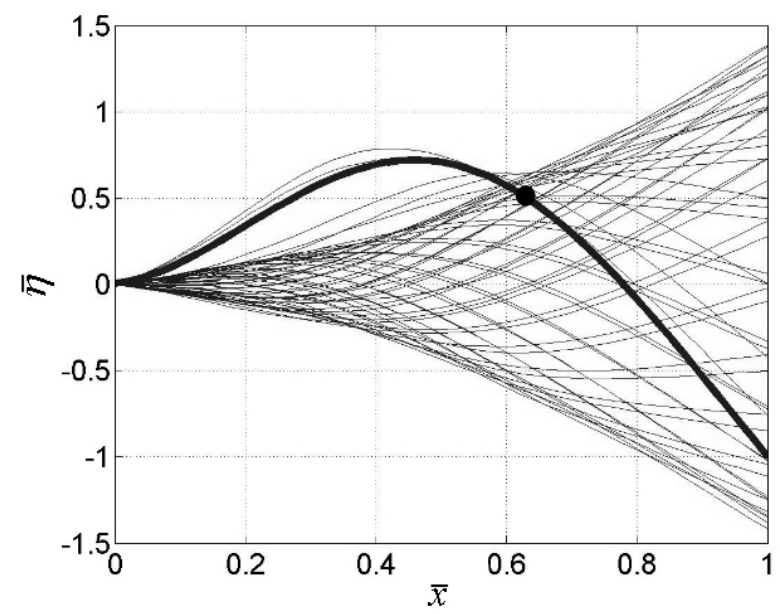

(b)

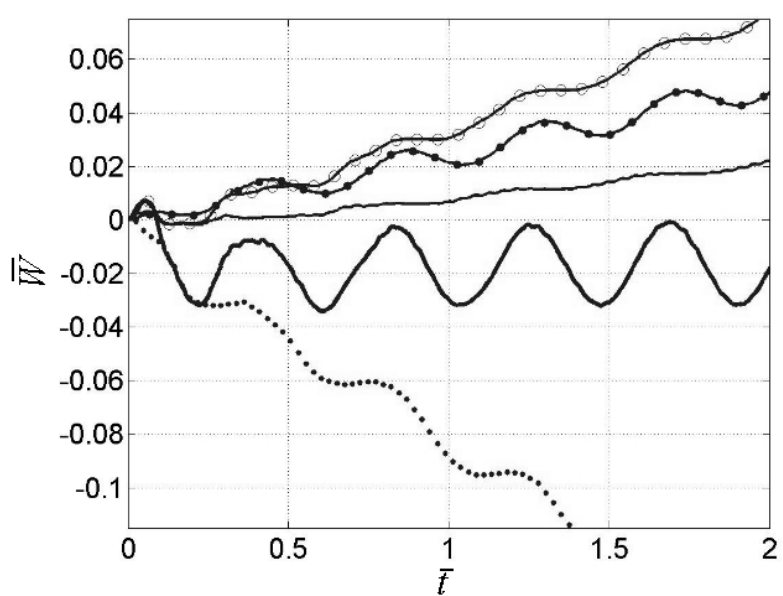

(c)

Figure 3: 


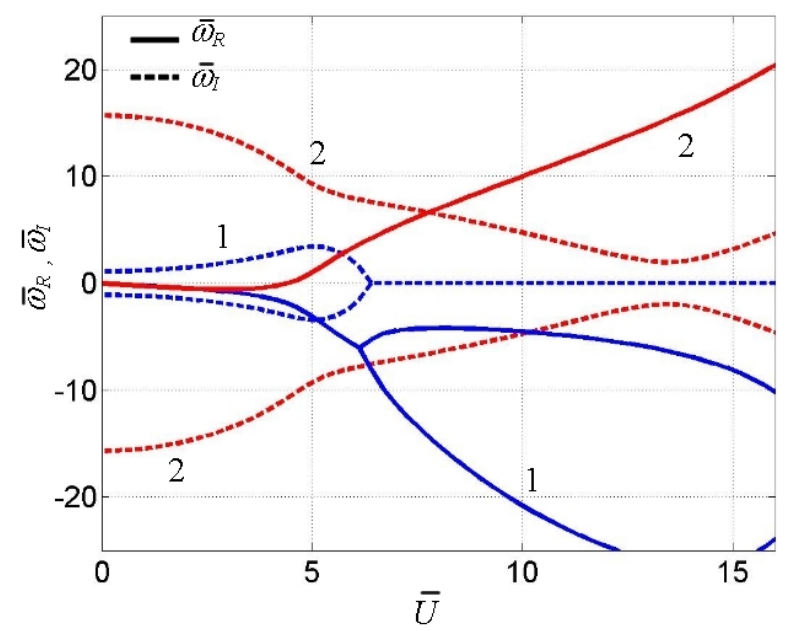

(a)

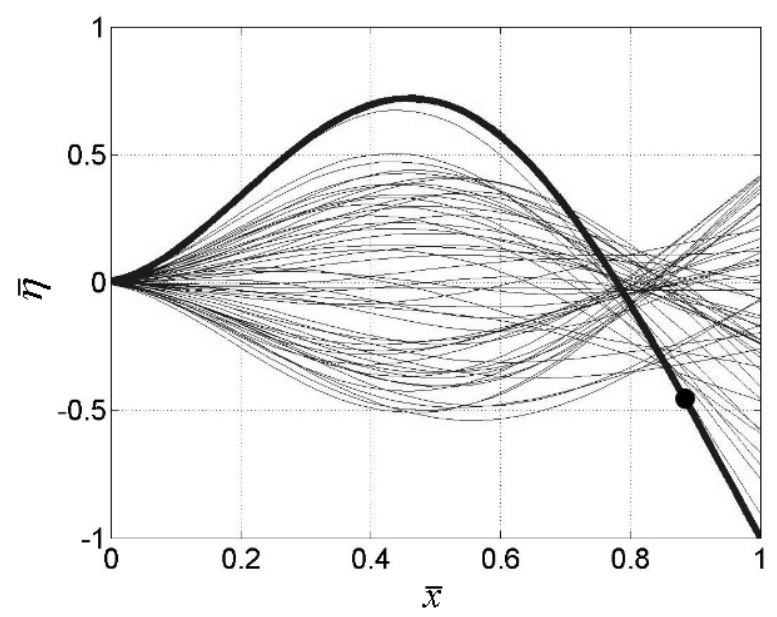

(b)

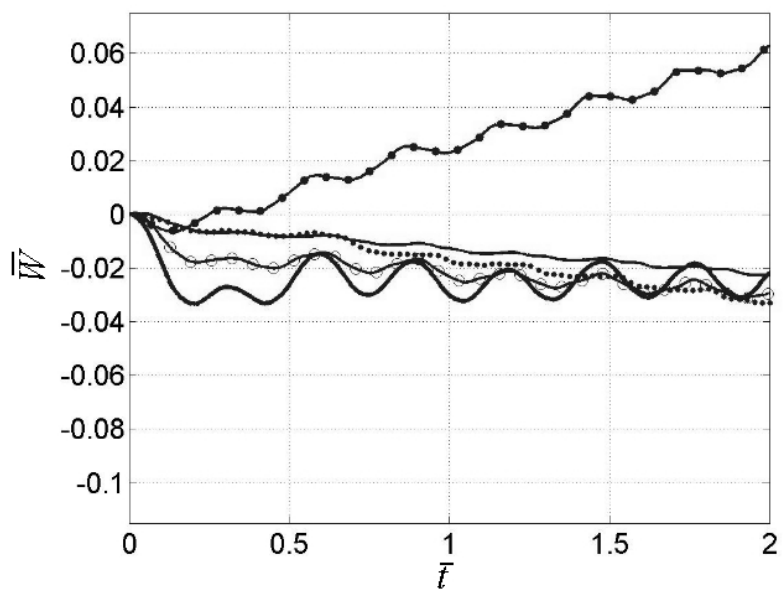

(c)

Figure 4: 


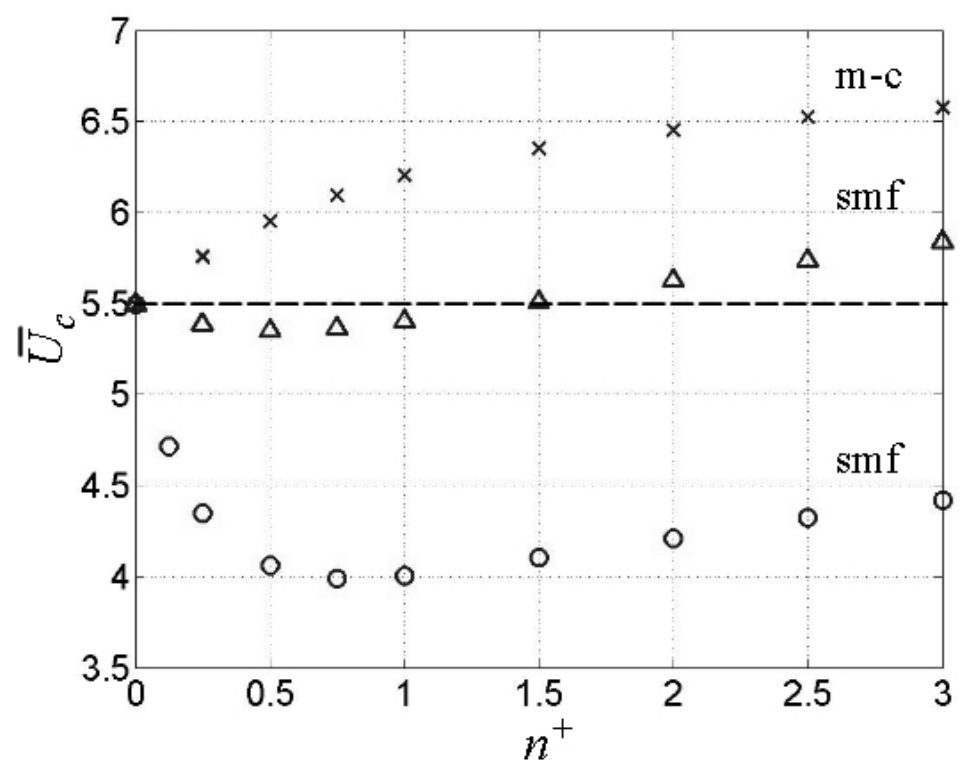

(a)

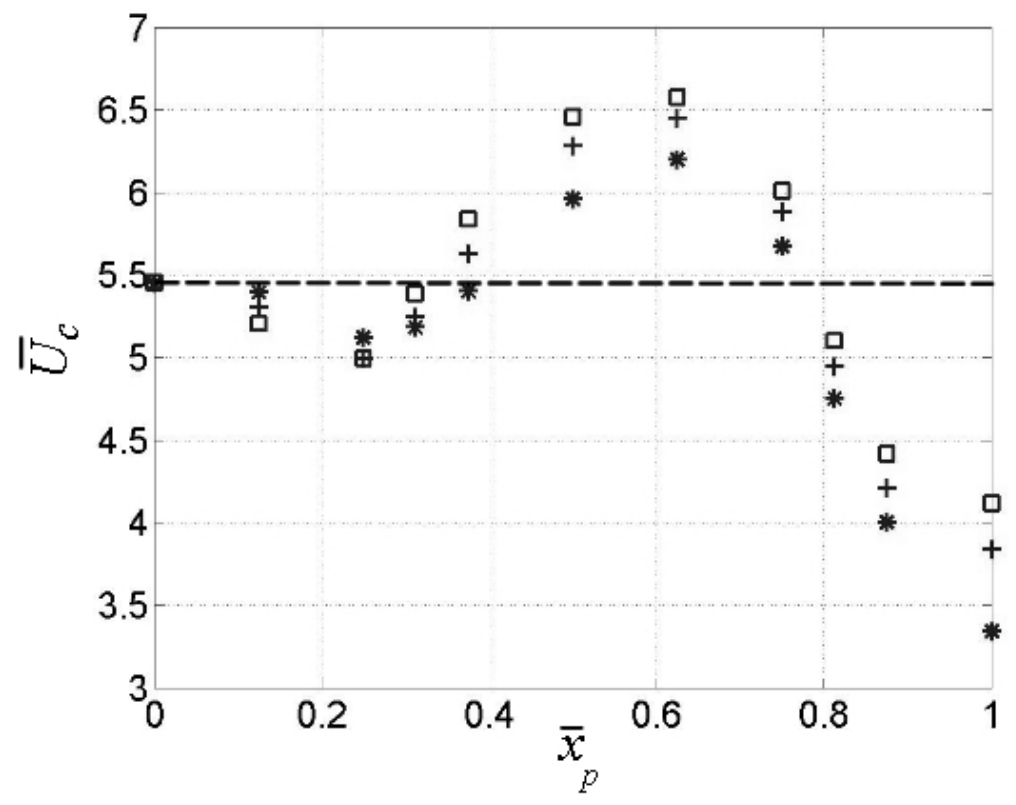

(b)

Figure 5: 


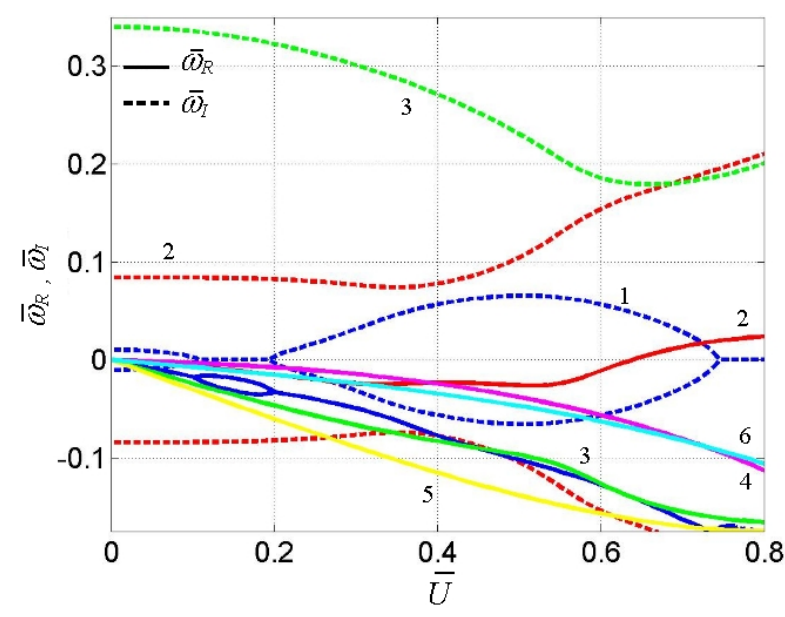

(a)

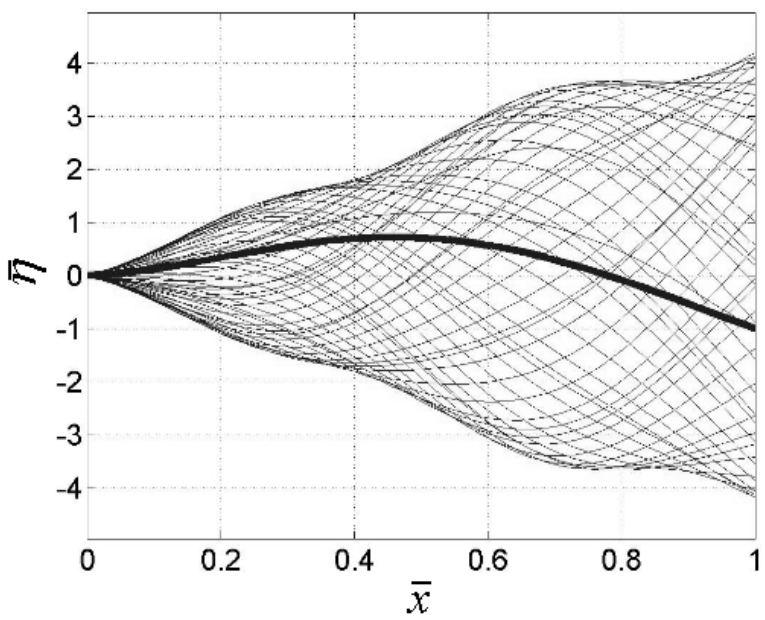

(b)

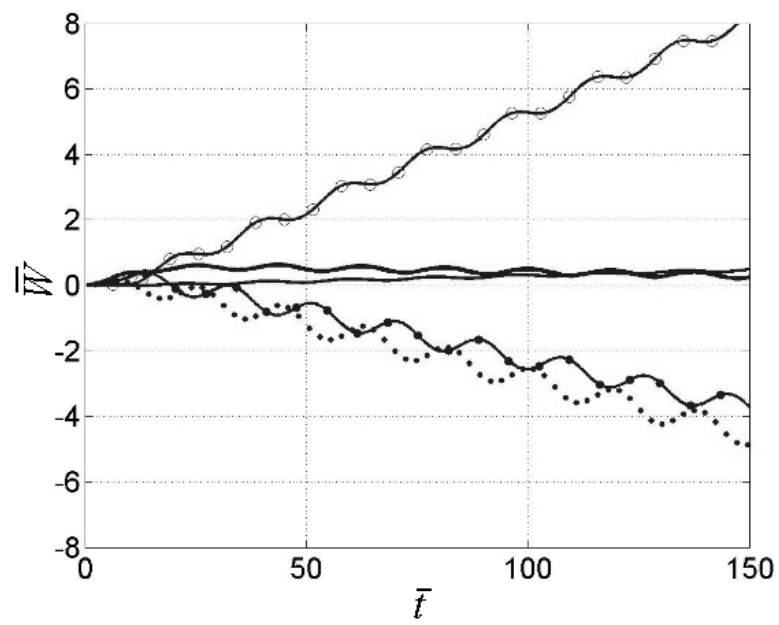

(c)

Figure 6: 


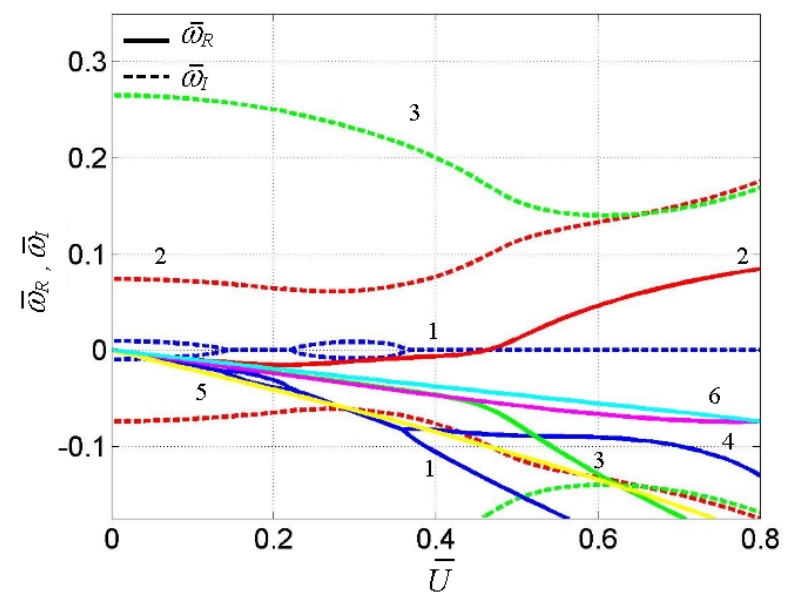

(a)

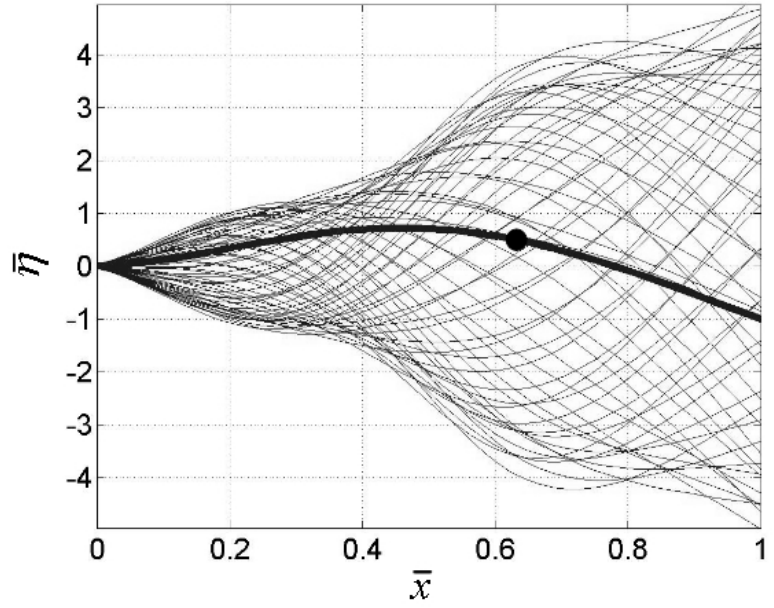

(b)

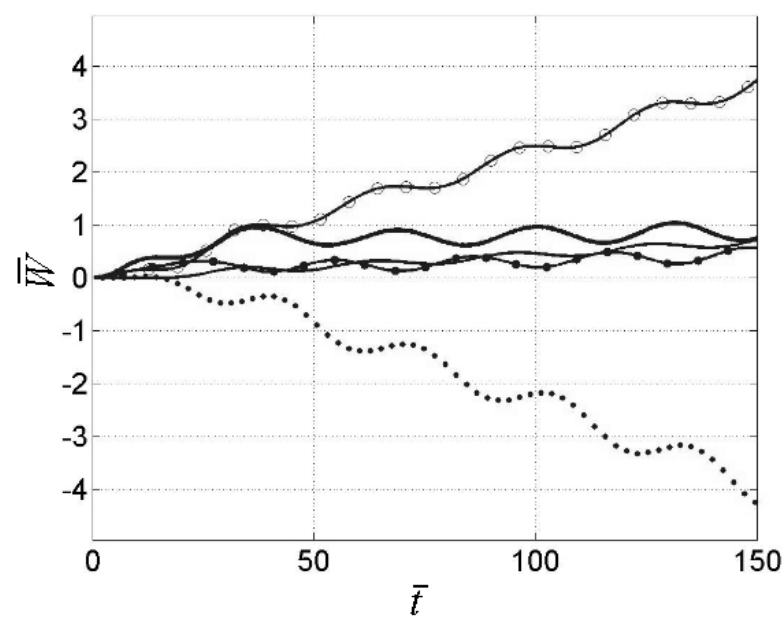

(c)

Figure 7: 


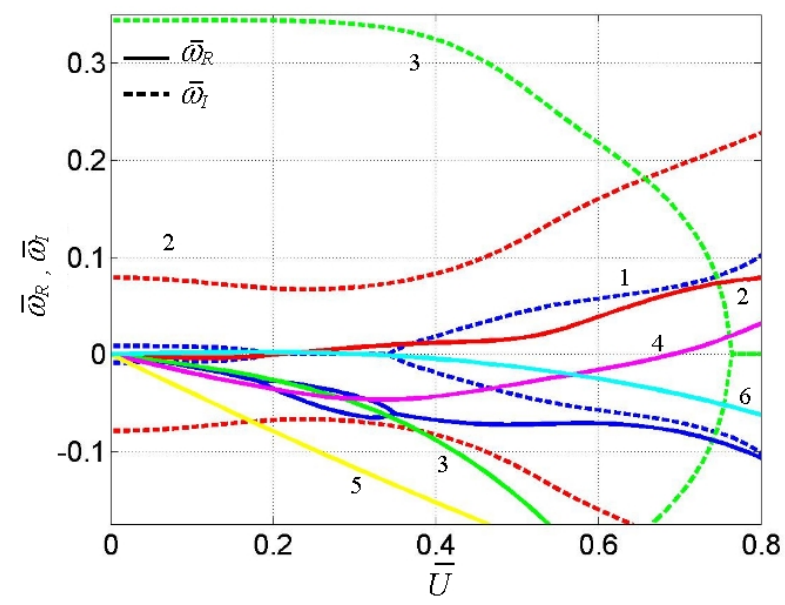

(a)

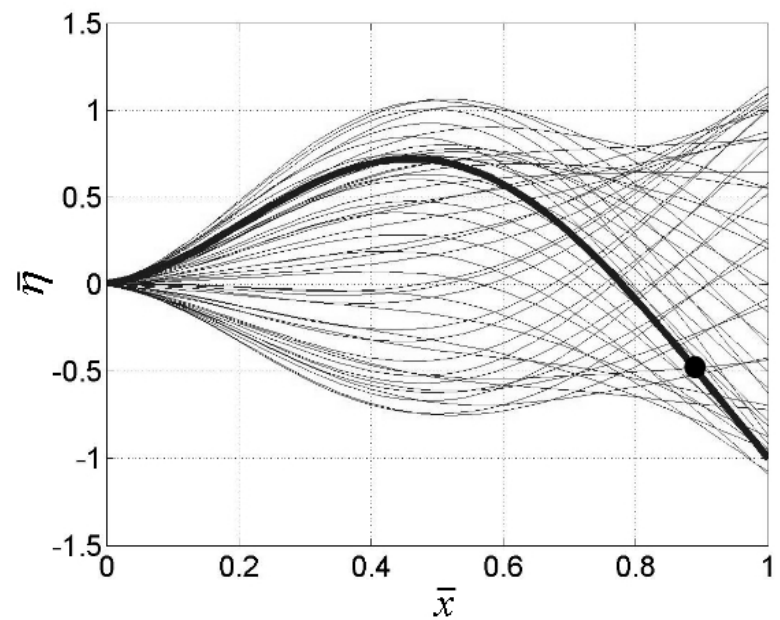

(b)

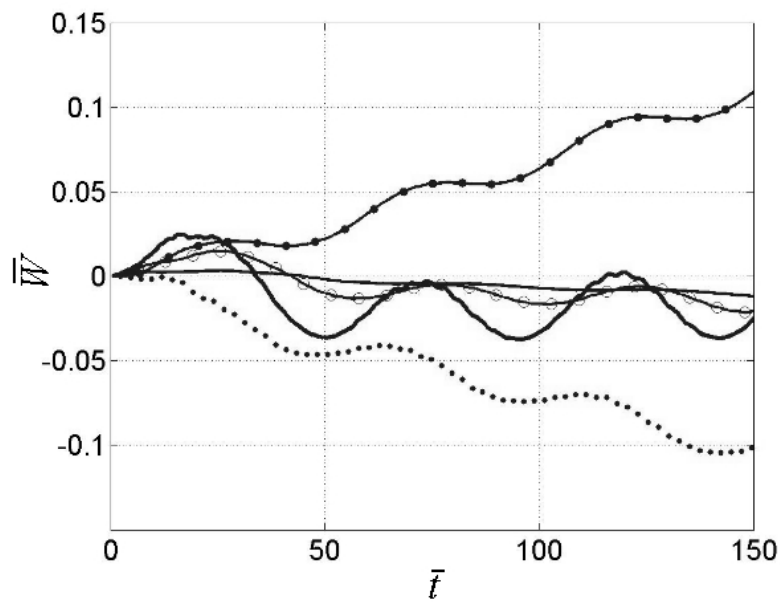

(c)

Figure 8: 


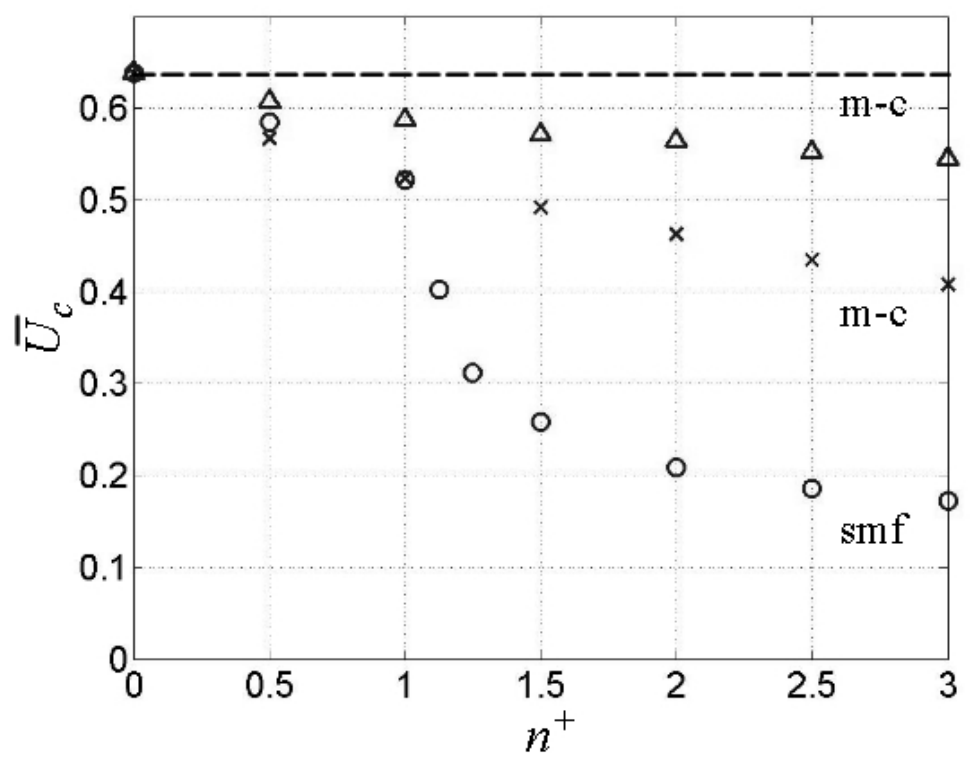

(a)

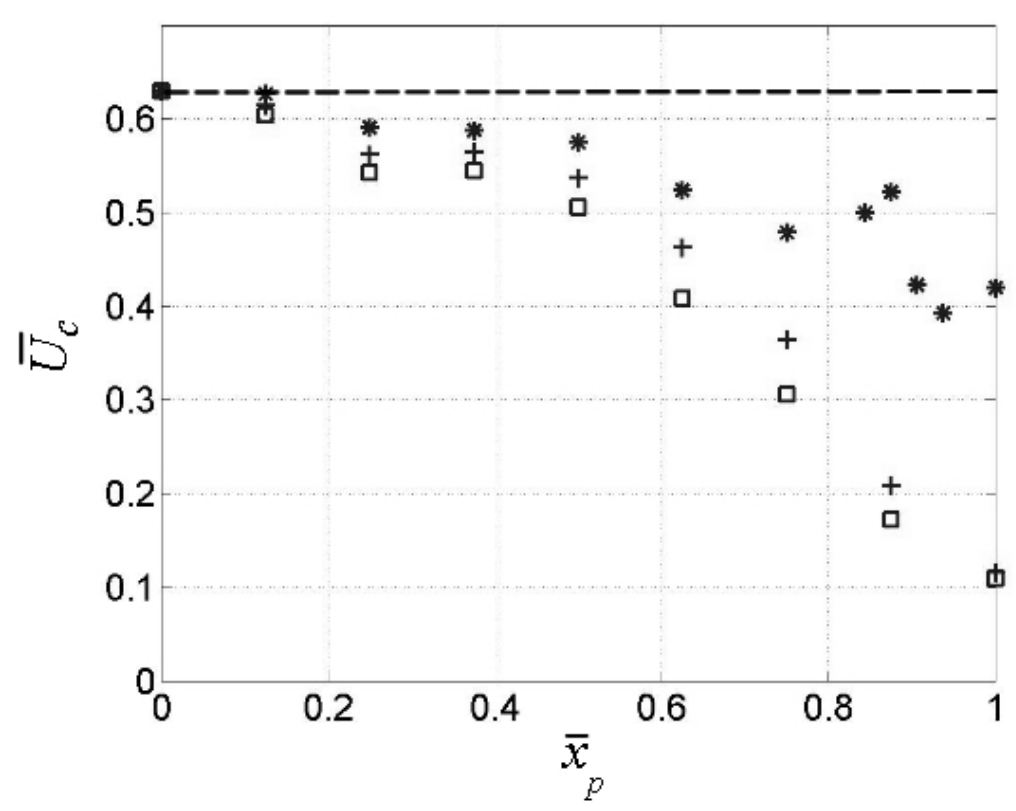

(b)

Figure 9: 